\title{
APPLICATIONS OF WAVELETS IN INDUCTION MACHINE FAULT DETECTION
}

\section{APLICACIONES DE WAVELETS EN LA DETECCIÓN DE FALLAS DE MÁQUINAS DE INDUCCIÓN}

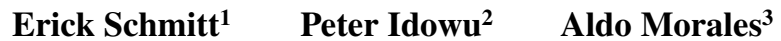 \\ Recibido 3 de diciembre de 2009, aceptado 26 de julio de 2010 \\ Received: December 3, 2009 Accepted: July 26, 2010
}

\begin{abstract}
RESUMEN
Este trabajo presenta un nuevo algoritmo basado en wavelets para la detección de fallas en máquinas de inducción de tres fases. Este nuevo método utiliza la desviación estándar de los coeficientes wavelet, que se obtiene de la descomposición de n-niveles de cada fase, para identificar fallas en el voltaje en una fase o fallas en la resistencia del estator en máquinas de inducción. El algoritmo propuesto puede funcionar independiente de la frecuencia de operación, tipo de falla y condiciones de carga. Los resultados muestran que este algoritmo tiene una mejor respuesta de detección que las técnicas basadas en la transformada de Fourier.
\end{abstract}

Palabras clave: Wavelets, detección de fallas, máquinas de inducción, transformada rápida de Fourier, detección temprana.

\begin{abstract}
This paper presents a new wavelet-based algorithm for three-phase induction machine fault detection. This new method uses the standard deviation of wavelet coefficients, obtained from n-level decomposition of each phase voltage and current, to identify single-phasing faults or unbalanced stator resistance faults in induction machines. The proposed algorithm can operate independent of the operational frequency, fault type and loading conditions. Results show that this algorithm has better detection response than the Fourier transform-based techniques.
\end{abstract}

Keywords: Wavelets, fault detection, induction machines, fast Fourier transform, early detection.

\section{INTRODUCTION}

Induction machines are among the most widely used devices in industrial processes today. They are generally viewed to be robust and well suited for a wide ranging applications. This increasing critical role in industrial processes underscores the level of attention given to early detection or diagnosis of potentially destructive faults, as well as the extensive research time devoted to the subject over the past decade.

Methods for prediction and detection of motor faults are extensively documented in the research literature; many of these methods use stator currents and voltage signals in some form along with signature algorithms to determine or predict fault conditions in an induction motor. A very organized summary of developments in motor signature analysis tools and techniques over the last two decades is presented by Benbouzid in [1]. Classical signature analysis techniques primarily use Fourier transform methods to examine current waveforms in details and then establish some criteria for classifying a range of rotor and stator faults. The trend in signature analysis is moving towards application of non-traditional computational techniques in the subject areas such as finite elements and more recently wavelet signal processing [1]-[4]. To contrast both approaches, induction motor fault diagnosis using Fourier techniques requires vast amount of data as established in [5]. Whereas, in [2] a Gaussian-enveloped wavelet was developed that searches for particular frequencies related to known faults, therefore improving fault-detection over the traditional Fourier method. However, note that machine's

\footnotetext{
1 E. Schmitt was with the Electrical Engineering Program, Penn State Harrisburg, 777 West Harrisburg Pike, Middletown, 17057 PA USA. E-mail: ejschmitt@gmail.com.

2 Electrical Engineering program, Penn State Harrisburg, 777 West Harrisburg Pike, Middletown, PA 17507, USA. E-mail: pbi1@psu.edu

3 Electrical Engineering program, Penn State Harrisburg, 777 West Harrisburg Pike, Middletown, PA 17507, USA. E-mail:awm2@psu.edu
} 
characteristic frequencies have to be known in advance and therefore bandpass filters with constant fractional bandwidth have to be designed for that particular machine. In [3], mechanical and electrical faults were detected by transients in the armature current and the fault detection algorithm was designed for brush d.c. windshield and fuel pump motors.

This paper presents a novel induction motor fault detection system that does not require a large amount of data such as in the Fourier analysis techniques. The method uses wavelet analysis to classify winding related motor problems such as open winding and winding resistance. Specifically standard deviation of wavelets coefficients were used for this purpose. After extensive simulations, it was determined that the reverse biorthogonal wavelet is the best one to extract features for our fault-detection algorithm. Note that in this algorithm, machine's characteristic frequencies do not have to be known in advance as in [2]. This algorithm also uses voltage and current information; unlike in [3] that use only armature current. In addition, in [3], the performance of the reverse biorthogonal wavelet was not investigated. Furthermore, the reduction of memory requirements allow the implementation of this system with lower cost hardware and permit the algorithm to be run in near real-time.

\section{OVERVIEW OF THE WAVELET TRANSFORM TECHNIQUE}

Fourier analysis techniques provide significant information on frequency components of signals under study, but offer no information regarding where a particular frequency was located in the time axis. In contrast, wavelet transforms offers time-frequency information of signals under study, thereby making wavelet transform methods more comprehensive than Fourier transforms in signal analysis.

Wavelet coefficients, at a first level of decomposition, are obtained from a signal under analysis by applying a mother wavelet. The process can be repeated if the mother wavelet is scaled and translated. The mother wavelet function (denoted by $\psi(t)$ ) and its scaling function (given as $\varphi(t))$ describe a family of functions which are required to satisfy a number of criteria $[7,8]$. It must have a zero mean denoted as in (1).

$$
\int_{-\infty}^{+\infty} \psi(t) d t=0
$$

In addition $\psi(t)$ must have a square norm of one as denoted in (2).

$$
\left.\int_{-\infty}^{+\infty} \psi(t)\right|^{2} d t=1
$$

These requirements are ensured by having a mother wavelet that is absolutely and square integrable. The mother wavelet forms a family of wavelets when the function is scaled and translated in the time domain. When a mother wavelet is translated by a factor of a and scaled by a factor of $b$, it can be expressed in a generic form as follows [7]:

$$
\psi_{a, b}(t)=\frac{1}{\sqrt{a}} \psi\left(\frac{t-b}{a}\right)
$$

The use of these wavelet functions provides a robust method of analyzing non-stationary signals to provide both frequency and time information. In practice, wavelet coefficients are obtained by a filter bank approach, with a low-pass filter and its complementary high-pass filter.

\section{APPLICATION OF WAVELETS IN INDUCTION MACHINE FAULT DIAGNOSIS}

The use of wavelets for induction machine fault detection is documented in various journals. They have been shown to yield satisfactory results for detecting electrical and mechanical faults [3]. Wavelet decomposition results in useful data contained in 'details' and 'approximate' parts as shown in the simplified block diagram of Figure 1. The 'approximation' signal can be further decomposed into a new set of 'approximation' and 'details' signals and continue until $n$ decomposition levels are obtained.

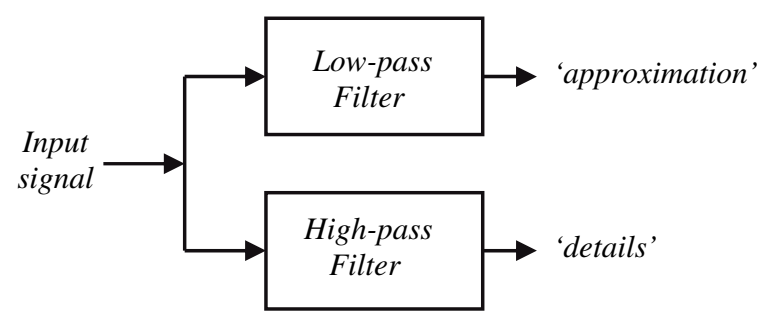

Figure 1. First level decomposition.

The 'details' signal contains high frequency information whereas the approximate part contains signal data with the low frequency components. Computing this decomposition to $n$ levels results in those higher detail parts being removed, thereby reducing the overall frequency characteristics of the resulting data. This implies that lower levels of decomposition provide detail data that contains the highest frequency components. For the induction machine signature analysis, the higher frequency wavelet components represent system noise or harmonics due to the input power inverter. Therefore decomposition levels higher than one are of interest in the technique presented in this paper. 
Fault patterns are obtained from the information yielded by the $n$-level wavelet decomposition through a variety of strategies, including filter banks and classification algorithms [8]. In this study a statistical analysis of the wavelet 'details' coefficients is used as the basis for fault detection. From the mean or standard deviation of the wavelet coefficients it could be inferred that the average magnitude of frequency components are present in the signal under analysis.

Each level of the signal detail coefficients provides frequency resolution that allows unique signature characteristics to be deduced. That is if the $n$-level detail coefficients are analyzed then each level represents the spatial information for a small range of frequencies. This allows the analysis of the frequency differences and their time location in the signal under analysis. In this paper, the standard deviation of the wavelets coefficients is used to identify frequency anomalies in a given time range in the input data set. The detection algorithm is discussed in details in the next section of this paper.

\section{PROPOSED ALGORITHM FOR FAULT DETECTION}

The algorithm presented in this paper uses the standard deviation of wavelet coefficients to detect single-phasing of supply (loose connection) and unbalanced stator resistance faults in three-phase machines. These types of faults tend to lead to a greater concentration of low and midrange frequency anomalies. An attempt to detect these faults with frequency domain techniques alone discards the cyclic nature of reoccurring patterns with each period. The analytical method proposed is independent of motor operating frequency. The basic values used for the decision process are referred to in this paper as the Standard Deviation of Wavelet Coefficients (SDWC). These values are calculated per phase by performing an $n$-level wavelet decomposition, then the standard deviation of these coefficients is obtained.

To detect the fault type, the maximum and minimum SDWC values are compared between the three phases. The ratio of these values is then used to detect the fault type. The ratio of the SDWC between phase 1 (minimum) and phase 2 (maximum) is given by $\beta_{1}^{2} \in[0,1]$. For example, if a sample set in phase A contains the greatest SDWC and phase B contains the lowest SDWC at half of the value of phase $A$, then this would produce a SDWC ratio of $\beta_{B}^{A}=0.5$. This value is used to test each data set to determine the presence of a winding fault. In the test cases presented in this paper, the optimal threshold values to determine the proper ratio for different faults were determined through experimentation. These ratio values will be denoted as $\beta^{\text {High }}, \beta^{\text {Mid }}$, and $\beta^{\text {Low }}$ for the three signature types detected. That is, $\beta^{\text {High }}$ is the SDWC range for a decision of no fault, $\beta^{\text {Mid }}$ is the range for a winding resistance fault, and $\beta^{L o w}$ is the range for open winding diagnosis. The decision process is shown functionally in the flow chart of Figure 2.

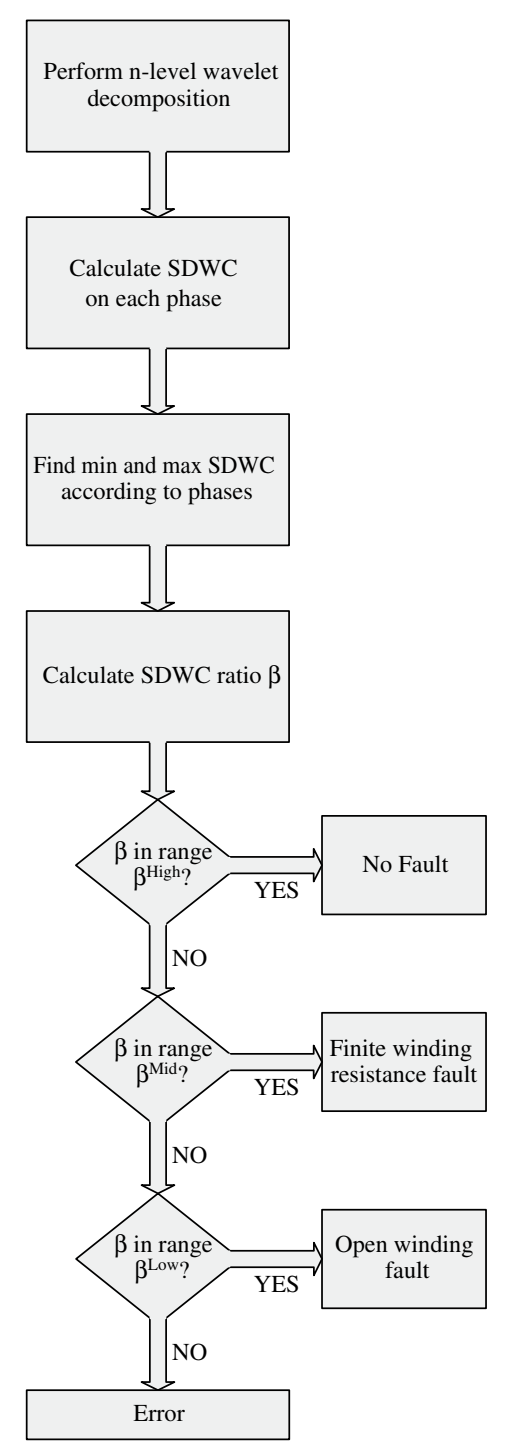

Figure 2. Flow chart of the algorithm.

This algorithm requires no learning and detects faults based on the differences between phases alone. The proposed diagnostic technique is tested using 63 different line-voltage and current measurements obtained from a set of identical 3-horsepower three-phase induction motors subjected to various types of faults. The Reverse Biorthogonal (rbio6) 
wavelet available in MATLAB ${ }^{\circledR}$ was used for testing the proposed algorithm.

\section{EXPERIMENTAL RESULTS AND FAULT DETECTION}

Data samples were gathered from input line voltages and currents of four different 3-horse-power induction motors fed through a three-phase inverter. The inverter offered the flexibility of adjusting machine supply frequency to 30,60 and $80 \mathrm{~Hz}$ to facilitate testing of the detection algorithm under varying machine supply frequencies. The electrical fault studies included unbalanced supply voltage, single-phasing of supply and unbalanced stator resistance. Measurements where recorded using a digital storage oscilloscope.

The faults were created by disconnecting the winding entirely or inserting a $15 \mathrm{Ohm}$ resistance on the phase. These faults were all produced on the induction machine phase B. A graphical user interface (GUI) was created in MATLAB $^{\circledR}$ to offer a convenient environment for users to run the detection algorithm and determine the fault type from test files generated following fault simulation. The GUI is shown in Figure 3.

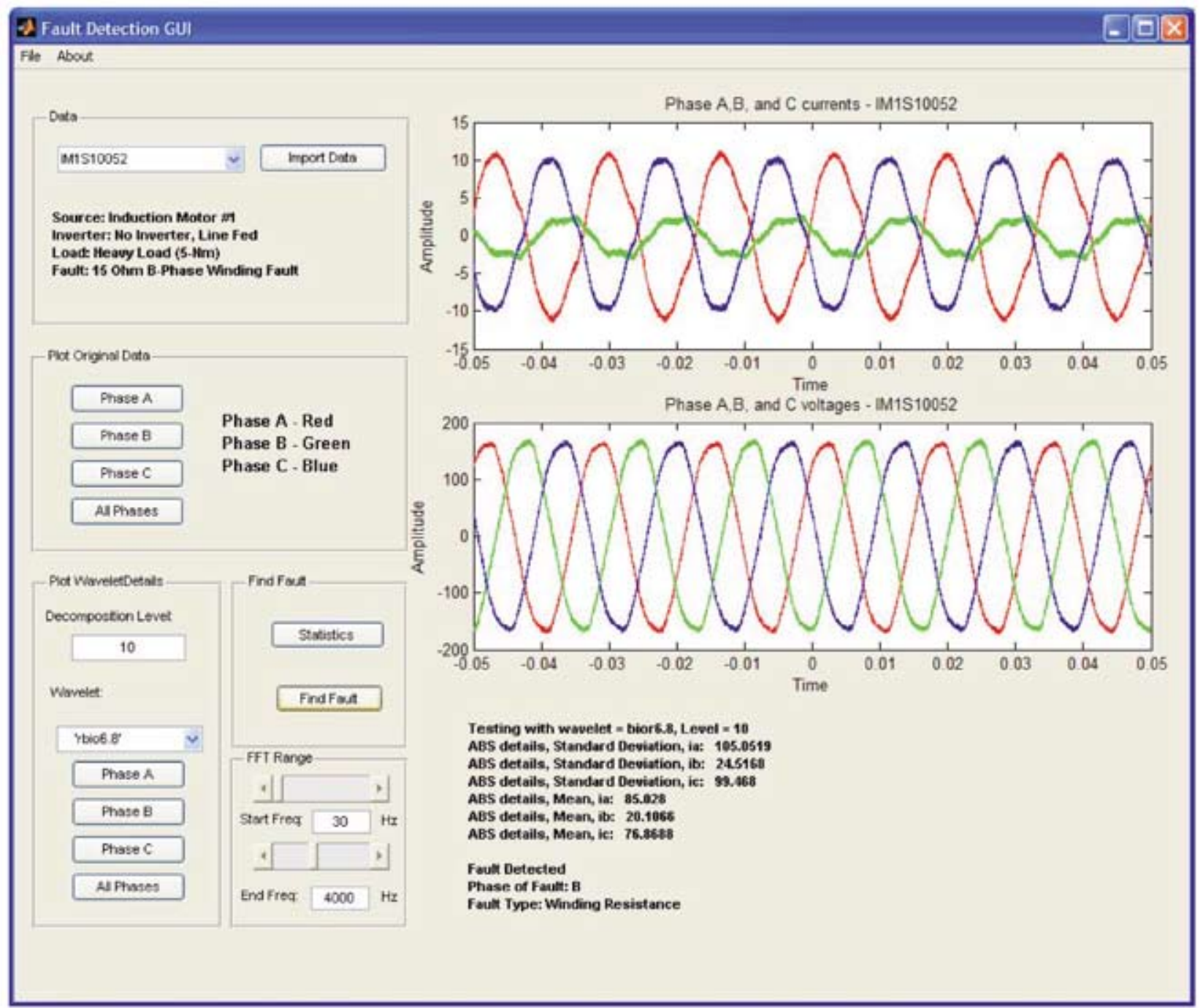

Figure 3. GUI interface for the fault detection algorithm.

The criteria used for fault detection was empirically determined using the input data set. The value used for $n$, the wavelet decomposition level, was ten, a value that will greatly magnify the frequency differences. This is considered a good choice because frequency differences for relatively low frequency systems such as the machines under study exist in lower bands. Decomposition coefficients at the lower levels simply compare electrical noise. The 
ranges used for $\beta^{\text {Low }}, \beta^{\text {Mid }}$, and $\beta^{\text {High }}$ where chosen to be $[0-0.1],[0.1-0.4]$, and $[0.4-1]$ respectively. Sixty-three cases (each case was stored in an excel file) with 10,000 data points each were used for testing this detection algorithm. The data was obtained at Penn State Harrisburg's Energy Conversion Laboratory. The data was wavelet-decomposed and the standard deviation of the wavelet coefficients for each phase obtained. The wavelet coefficients for each phase is summarized and shown graphically in Figure 4.

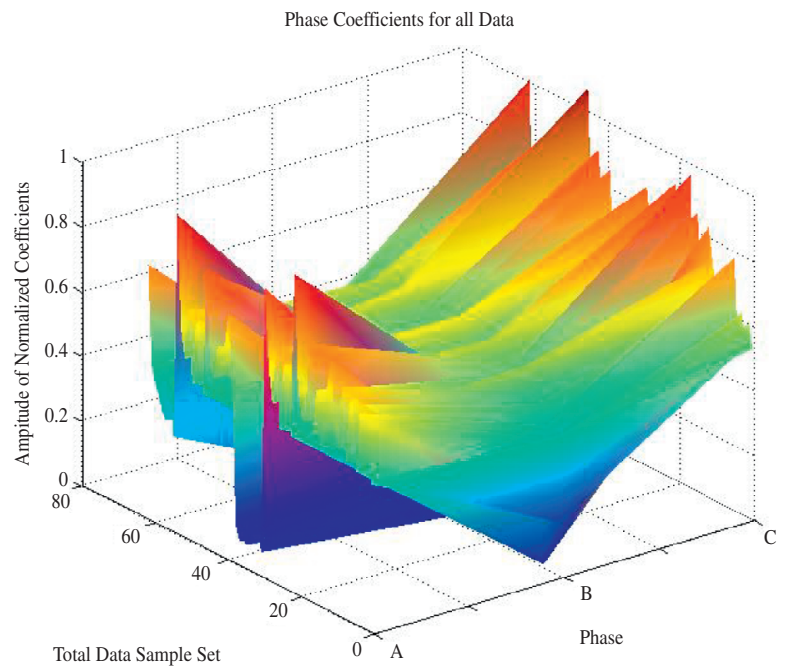

Figure 4. Normalized Standard deviation of Wavelet coefficients for all 63 data samples.

Figure 4 shows the values of the normalized standard deviation of the wavelet coefficients for each phase. At first observation, the data seems arbitrary. Since the data set contains many sets with faults, therefore it is expected a trend could be seen in phase B. This however shows very little information about the type of fault. The data was then run through the algorithm to detect the fault conditions. The faults were detected correctly for every case. The algorithm was set up to routinely sort the different samples based on fault type. This allowed for new plots to be constructed showing the similarities between data sets processing similar faults. Figures 5, 6 and 7 shows the cases with no fault, winding resistance fault and open winding faults respectively. The "Data Sample Set" axis of these figures show how many files were used in each case. For instance, Figure 5 displays that 32 files were used for the proposed algorithm, containing a total of 32,000 data points. Figures 6 and 7 display similar attributes. These plots clearly show the trends of the input data and provide enough feature information of the type of fault to be detected.

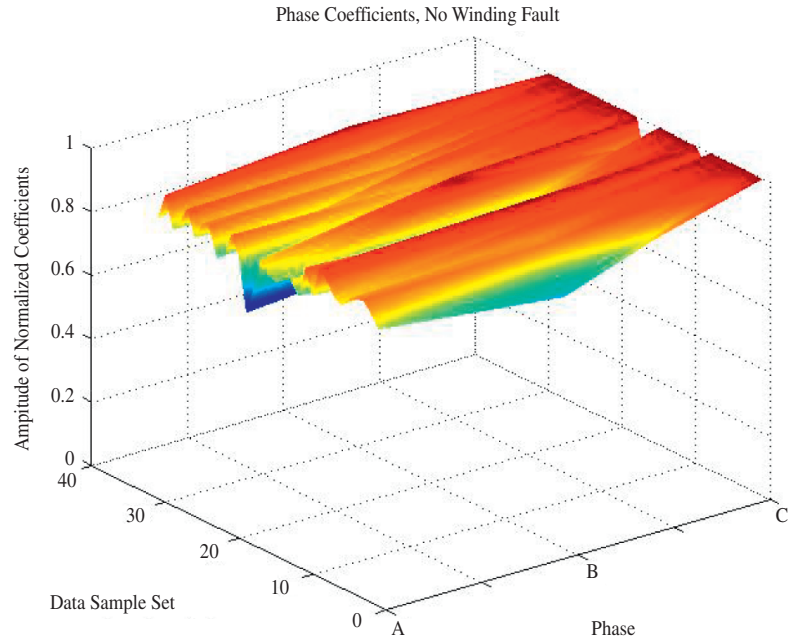

Figure 5. Wavelet coefficients showing no winding fault (32 files out of 63).

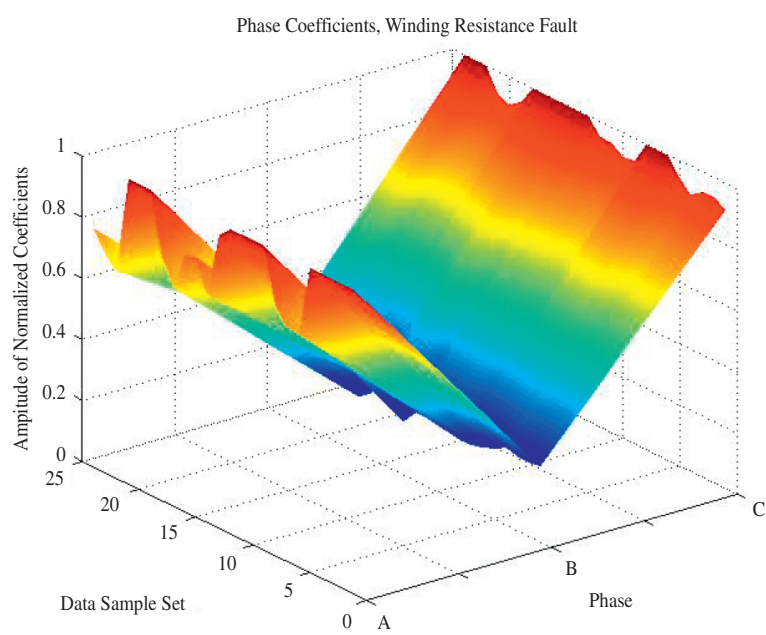

Figure 6. Wavelet coefficients showing winding resistance fault (24 files out of 63).

The data could also be presented looking at the criteria originally used to determine the fault type, which is the ratio of the minimum to the maximum standard deviation of the wavelet coefficients. Figure 8 shows the data samples as they were analyzed where the no fault and the two fault conditions clearly demonstrate distinct behavior. The blue bars show the no fault condition, the green bars illustrate winding resistance faults and the smaller red (difficult to see at, the bottom of Figure 8) bars depict the open winding resistance fault. Notice that the fault conditions yield a large difference in coefficients, and therefore smaller ratios (shorter bars). Again this data 
clearly shows that a correct detection can be obtained by using this algorithm.

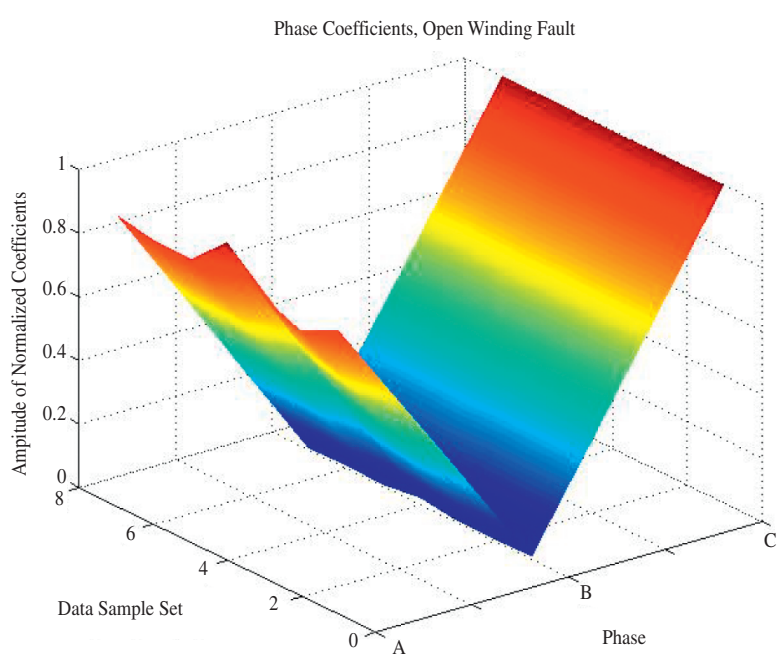

Figure 7. Wavelet coefficients showing open winding fault (7 files out of 63).

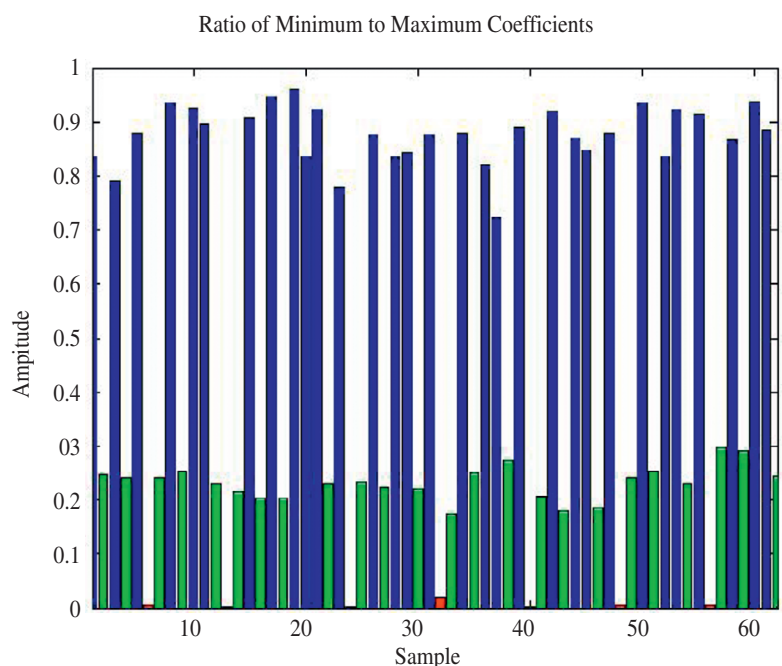

Figure 8. Relative ratio between phases of standard deviation of wavelet coefficients.

\section{COMPARISON WITH FOURIER TRANSFORM TECHNIQUES}

The field of induction machine fault detection has been generally based on the use of the Fast Fourier Transform (FFT). This technique is well established and greatly researched in the literature, see [9] for an overview. The algorithm proposed here has key advantages over classic FFT solutions. The use of FFT-based motor fault detection requires that frequency resolution be very good, generally less than $1 \mathrm{~Hz}$. To achieve this resolution the data set used must be very large and therefore requires a large amount of memory for processing. The algorithm presented here performs without that requirement, in fact attempting an FFT-based fault detection algorithm on the same data set used in the wavelet-based algorithm, yields inconclusive results. This is illustrated in Figure 9 where the data presented previously (with a known fault) has very poor frequency resolution around.

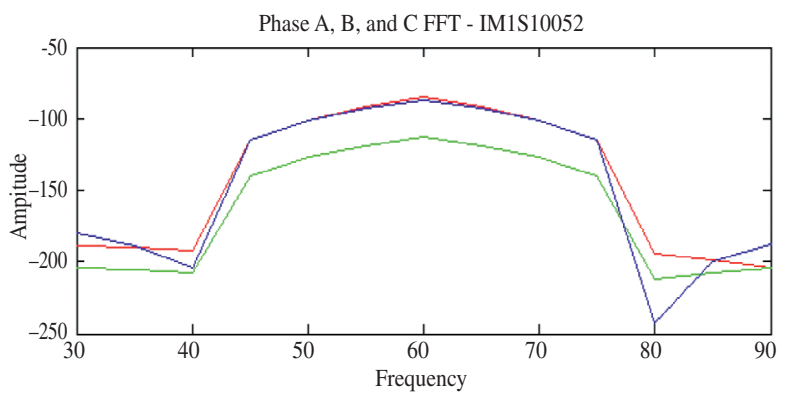

Figure 9. FFT of motor current with known fault condition.

The results obtained in section 5 and in this section, and the amount of data analyzed, provide validity of the proposed algorithm. In particular, Figures 5, 6,7, and 8 clearly show the distinctive features of the normalized standard deviation that were used as signal signature for this method. Note, once again that the decision algorithm is frequency-independent. We highlight that the fault detection software not only has been successfully implemented but also used in a teaching environment for introducing wavelet applications in signature analysis in an undergraduate energy conversion course [11].

\section{CONCLUSIONS}

Wavelet decomposition is a superior method of signal analysis in time varying situations due to spatial data retention. Analysis using wavelets produces both frequency and spatial information providing a robust solution for motor fault detection. Induction machines account for the majority of industrial equipment in use today and proper operation is of utmost importance.

The fault detection algorithm discussed in this paper could identify a fault present in motor that is still operational, therefore can provide preventive maintenance schedule. Faults such as those discussed in this paper should be detected before they cause the machine to completely fail and avoid total system degradation. Detecting these faults in a timely manner is vital to maintaining a properly functional system. The proposed method offers a reliable solution for detecting the fault types of interest. This algorithm was tested on over sixty data samples, on 
four different induction machines and detected faults with $100 \%$ reliability. This algorithm is independent of operational frequency, fault type, and loading. In addition, the algorithm also requires no training and provides a more robust solution compared to systems simply comparing characteristics to known healthy values. That allows this system to work for many different motors and account for normal motor wear that may cause false positive detection in some algorithms since only the differences between windings is used as a detection criteria.

\section{ACKNOWLEDGMENT}

The authors would like to thank the anonymous reviewers for their helpful comments.

Preliminary version of this paper was presented at the 2008 American Society for Engineering Education Conference, Pittsburgh, Pennsylvania, USA.

\section{REFERENCES}

[1] M. Benbouzid. "A review of induction motors signature analysis as a medium for faults detection". IEEE Transactions on Industrial Electronics. Vol. 47, Issue 5, pp. 984-993. October, 2000.

[2] T. Chow and H. Shi. "Induction machine fault diagnostic analysis with wavelet technique". IEEE Transactions on Industrial Electronics. Vol. 51, Issue 3, pp. 558-565. June, 2004.

[3] W.G. Zanardelli, E.G. Strangas, H.K Khalil and J.M. Miller. "Wavelet-based methods for the prognosis of mechanical and electrical failures in electric motors". Mechanical Systems and Signal Processing. Vol. 18, Issue 2, pp. 411-426. March, 2005.
[4] G.K. Singh and S. Kazzaz. "Vibration signal analysis using wavelet transform for isolation and identification of electrical faults in induction machine". Electric Power Systems Research. Vol. 68, Issue 2, pp. 119-136. February, 2004.

[5] E.A. Ebrahim and N. Hammad. "Fault analysis of current-controlled PWM-inverter fed inductionmotor drives". Proceedings of the IEEE International Conference on Properties and Applications of Dielectric Materials. Vol. 3, pp. 1065-1070. 2003.

[6] M. Benbouzid and G. Kliman. "What stator current processing-based technique to use for induction motor rotor faults diagnosis?". IEEE Transactions on Energy Conversion. Vol. 18, Issue 2, pp. 238244. June, 2003.

[7] G. Strang and T. Nguyen. "Wavelets and Filters Banks". Wellesley-Cambridge Press. Wellesley, MA, USA. 1996.

[8] M. Vetterli and J. Kovacevic. "Wavelets and Subband Coding”. Prentice-Hall. Upper Saddle River, New Jersey, United States. April, 1995.

[9] Z. Zhengping, R. Zhen, and H. Wenying. "A novel detection method of motor broken rotor bars based on wavelet ridge". IEEE Transactions on Energy Conversion. Vol. 18, Issue 3, pp. 417-423. September, 2003.

[10] W.T. Thomson and M. Fenger. "Current signature analysis to detect induction motor faults". IEEE Industry Applications Magazine. Vol. 7, Issue 4, pp. 26-34. July/August, 2001.

[11] P. Idowu, J. Atiyeh, E. Schmitt and A. Morales. "MATLAB® Tool for Introducing Basics of Induction Motor Signature (IMCS) Analysis". Accepted for publication. International Journal of Electrical Engineering Education. July 29, 2009. 